\title{
Search for exotic production of top quarks decaying into same-sign leptons at $13 \mathrm{TeV}$ with the ATLAS detector
}

\section{S. Berlendis*}

LPSC, CNRS/IN2P3, Université Grenoble-Alpes, Grenoble, France

E-mail: simon.paul.berlendisecern.ch

On behalf of the ATLAS Collaboration

\begin{abstract}
The signature of same-sign leptons and b-jets is a golden channel in the search for exotic production of top quarks. One of the main sources of background of same-sign leptons come from detector effects: leptons with mis-reconstructed charge and fake/non-prompt leptons. Sophisticated data-driven techniques are needed to accurately estimate them. A description of the analysis strategy and of the different techniques used to estimate the background are given here. Limits extracted for various models beyond the Standard Model are shown with the 2015 proton-proton collisions data recorded at $\sqrt{s}=13 \mathrm{TeV}$ with the ATLAS detector at the Large Hadron Collider.
\end{abstract}

Fourth Annual Large Hadron Collider Physics

13-18 June 2016

Lund, Sweden

\footnotetext{
* Speaker.
} 


\section{Motivation and strategy}

The analysis of events containing same-sign leptons and $b$-jets is motivated by the search for physics beyond the Standard Model (BSM) predicting exotic productions of top quarks. This final state is a striking signature in the ATLAS detector [1] as it has a relatively low background from the Standard Model (SM). The analysis of this final state performed with the data recorded at $13 \mathrm{TeV}$ with an integrated luminosity of $3.2 \mathrm{fb}^{-1}$ [2] is presented here.

Three BSM models are considered in this analysis. The vector-like quarks model [3] predicts the pair production of vector-like quark (VLQ) decaying into a top quark and an extra boson. Three signals are considered: $T_{5 / 3} \bar{T}_{5 / 3}$ pairs with $T_{5 / 3} \rightarrow t W, T \bar{T}$ pairs with $T \rightarrow b W / t Z / t H$ and $B \bar{B}$ pairs with $B \rightarrow t W / b Z / b H$. The extra-dimension model with two universal extra dimensions under the real projective plane geometry (2UED/RPP) [4] predicts Kaluza-Klein excitations of the photon $\left(A_{\mu}^{(1,1)}\right)$ decaying into top quarks. This model is parameterized by the two radii $R_{4}$ and $R_{5}$ of the extra dimensions. The contact interaction model [5] predicts an effective four top quarks interaction with the coupling strength $C_{4 t} / \Lambda^{2}$. Examples of Feynman diagrams for these three models are presented in Figure 1.

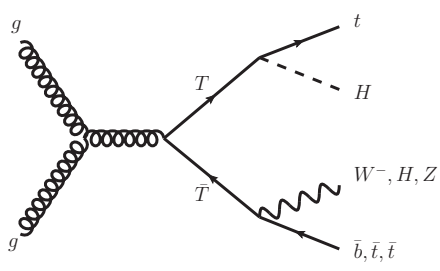

(a)

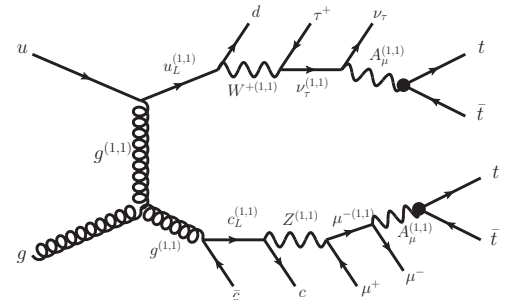

(b)

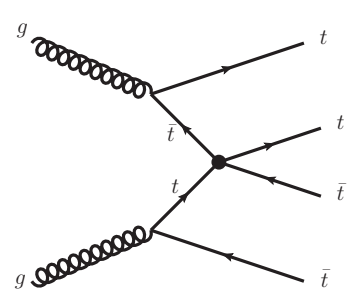

(c)

Figure 1: Examples of Feynman diagrams of (a) pair production of vector-like quarks, and four-top production (b) in an extra-dimension model and (c) by contact interaction.

A similar analysis was performed at $8 \mathrm{TeV}$ and a modest excess was found in two signal regions [6]. The global significance of this excess was estimated to be around $2.5 \sigma$. The analysis at $13 \mathrm{TeV}$ allows to check the physical or the statistical nature of this excess.

For this reason, the selection strategy is kept the same as in the $8 \mathrm{TeV}$ analysis. The definition of the signal regions are shown in Table 1. They are based on selecting same-sign dilepton or trilepton events with different requirements on the missing transverse momentum $E_{\mathrm{T}}^{\text {miss }}$, the jet activity $H_{\mathrm{T}}=\sum p_{\mathrm{T} \text { jets }}$ and the b-jet multiplicity $N_{b}$.

\section{Background estimation}

The background contribution from SM processes leading to true same-sign leptons is modeled with Monte-Carlo generators. The dominant processes are the production of a pair of top quarks with extra boson(s) $(t \bar{t}+W / Z / W W)$ and the production of dibosons $(V V)$. The background also includes the rare production of tribosons $(V V V)$, higgs boson $(V H / t \bar{t} H)$ and multi-tops (three and four top quarks). 


\begin{tabular}{c|c|c|c}
\hline \hline \multicolumn{3}{c|}{ Definition } & Name \\
\hline$e^{ \pm} e^{ \pm}+e^{ \pm} \mu^{ \pm}+\mu^{ \pm} \mu^{ \pm}+e e e+e e \mu+e \mu \mu+\mu \mu \mu, N_{\text {jets }} \geq 2$ \\
\hline \multirow{3}{*}{$400<H_{\mathrm{T}}<700 \mathrm{GeV}$} & $N_{b}=1$ & \\
\cline { 4 - 4 } & $N_{b}=2$ & $E_{\mathrm{T}}^{\text {miss }}>40 \mathrm{GeV}$ & SR0 \\
\cline { 4 - 4 } & $N_{b} \geq 3$ & & SR1 \\
\cline { 4 - 4 }$H_{\mathrm{T}} \geq 700 \mathrm{GeV}$ & $N_{b}=1$ & $40<E_{\mathrm{T}}^{\text {miss }}<100 \mathrm{GeV}$ & SR3 \\
\cline { 2 - 4 } & & $E_{\mathrm{T}}^{\text {miss }} \geq 100 \mathrm{GeV}$ & SR4 \\
\cline { 2 - 4 } & $N_{b}=2$ & $40<E_{\mathrm{T}}^{\text {miss }}<100 \mathrm{GeV}$ & SR5 \\
\cline { 2 - 4 } & $N_{b} \geq 3$ & $E_{\mathrm{T}}^{\text {miss }} \geq 100 \mathrm{GeV}$ & SR6 \\
\cline { 2 - 4 } & $E_{\mathrm{T}}^{\text {miss }}>40 \mathrm{GeV}$ & SR7 \\
\hline
\end{tabular}

Table 1: Definition of the different signal regions. The jet and $b$-jet multiplicities are denoted by $N_{\text {jets }}$ and $N_{b}$, respectively.

Opposite-sign dilepton events can be reconstructed as same-sign dilepton events if one of the leptons has a mis-reconstructed charge. This charge mis-identification (Charge mis-ID) is negligible for muons. For electrons, it can be due to a wrong charge reconstruction from the tracker detector or due to a photon conversion $\left(e \rightarrow e \gamma, \gamma \rightarrow e^{+} e^{-}\right)$. This background is estimated by weighting opposite-sign lepton events using the charge flip rates for each electron. The latter is estimated as a function of the kinematics of the electron $\left(p_{\mathrm{T}_{e}}\right.$ and $\left.\eta_{e}\right)$ in $Z \rightarrow e e$ enriched data events by minimizing a Poisson likelihood. A systematic uncertainty of $20-25 \%$ is assigned to this background to take into account the uncertainty on the measured charge flip rates.

The fake leptons are defined as non-prompt leptons or as jets mis-identified as lepton. This background is estimated using a matrix method combined with a likelihood approach. In this method, a loose region enriched in fake leptons is defined using a looser lepton identification criterion (loose) compared to the nominal one (tight). The fake background is estimated by minimizing a Poisson likelihood with the number of loose and tight leptons in the loose region. The real (fake) efficiency $\varepsilon_{r(f)}$ is defined as the probability of one real (fake) lepton to be tagged as tight. It is estimated in data in a single-lepton control region enriched in real (fake) leptons as a function of the kinematics of the lepton $\left(p_{\mathrm{T} \ell}, \eta_{\ell}\right.$ and $\left.\Delta R\left(\ell, 1^{s t} \mathrm{jet}\right)\right)$ and of the jet multiplicities $\left(N_{\mathrm{jets}}\right.$ and $\left.N_{b}\right)$. A systematic uncertainty of $52 \%$ is assigned to this background to take into account the uncertainty on the measured real/fake efficiencies.

The true same-sign leptons, the charge mis-identification and the fake leptons represent respectively $39 \%, 34 \%$ and $27 \%$ of the total background in all the signal regions combined.

\section{Control regions}

The background estimate is validated in different control regions orthogonal to the signal regions. One of them is defined by selecting same-sign dilepton/trilepton events with a lower jet activity $\left(100 \mathrm{GeV}<H_{\mathrm{T}}<400 \mathrm{GeV}\right)$ and at least one b-jet. Figure 2 shows three distributions obtained in this control region. The background estimate is found to be in agreement with the data within the total uncertainty in all the control regions. 


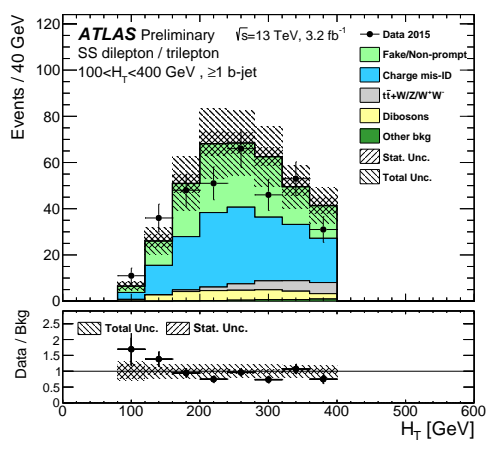

(a)

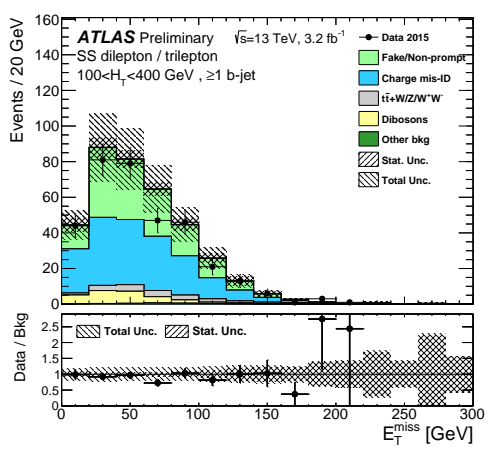

(b)

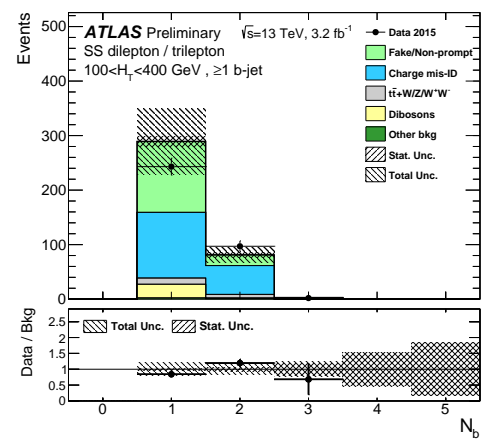

(c)

Figure 2: Distribution of the (a) jet activity $H_{\mathrm{T}}$, (b) missing transverse momentum $E_{\mathrm{T}}^{\text {miss }}$, and (c) b-jet multiplicity $N_{b}$ in the control region defined as $100 \mathrm{GeV}<H_{\mathrm{T}}<400 \mathrm{GeV}$ and $N_{b} \geq 1$. The bottom panels display the ratios of data to the total background prediction.

\section{Results and interpretation}

The comparison between the data and the background estimate for all signal regions is shown in Figure 3. In all signal regions, no significant excess is found. The modest excess found at $8 \mathrm{TeV}$ in SR6 and SR7 as defined in Table 1 is therefore not confirmed by the first $3.2 \mathrm{fb}^{-1}$ data at $13 \mathrm{TeV}$.

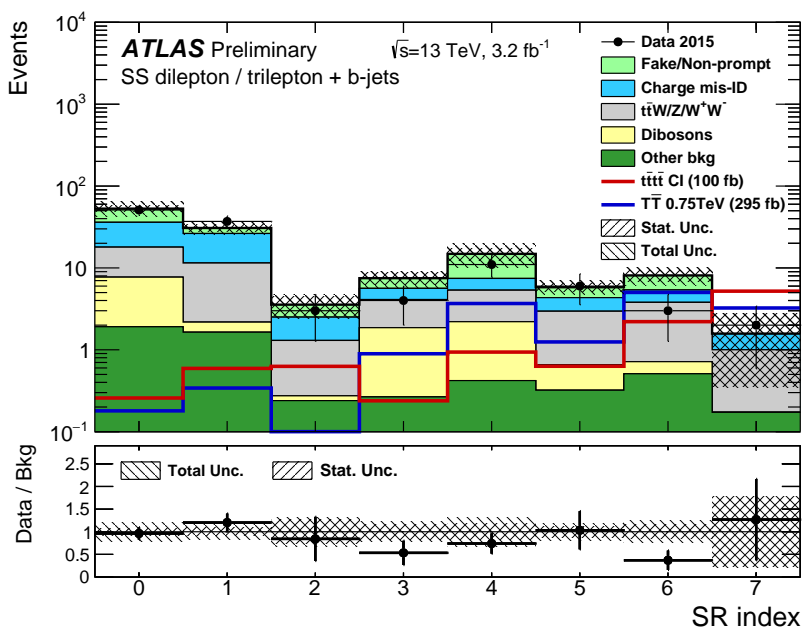

Figure 3: Data and expected background yields in the signal regions defined in Table 1. The bottom panel displays the ratios of data to the total background prediction.

Limits at $95 \%$ confidence level (CL) are set on each considered model by combining all the signal regions and by using a profile log-likelihood ratio as statistical test. Figure 4 shows different limits on the BSM models described in Section 1. By comparison, a similar analysis performed at $13 \mathrm{TeV}$ by the CMS collaboration [7] set an observed (expected) exclusion of $960 \mathrm{GeV}$ (900 GeV) on the $T_{5 / 3}$ mass against $990 \mathrm{GeV}(920 \mathrm{GeV})$ in this analysis. 


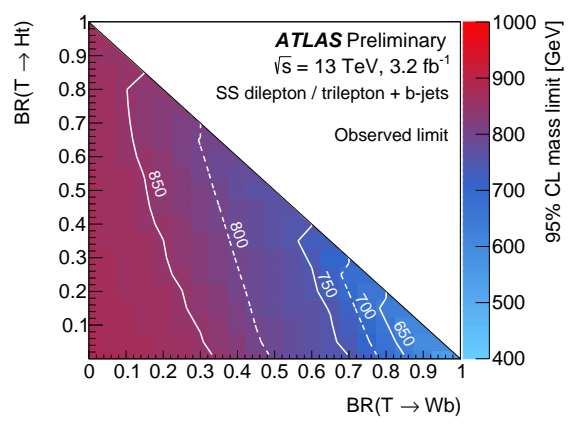

(a)

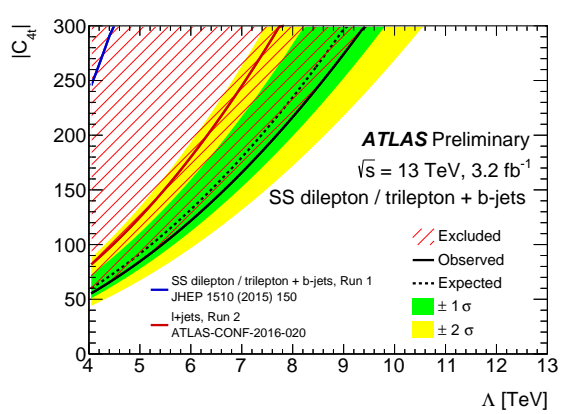

(c)

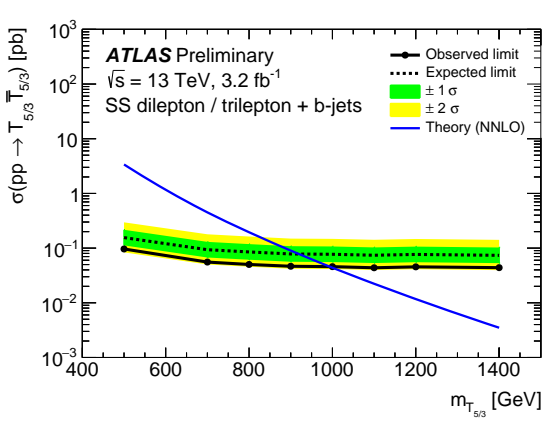

(b)

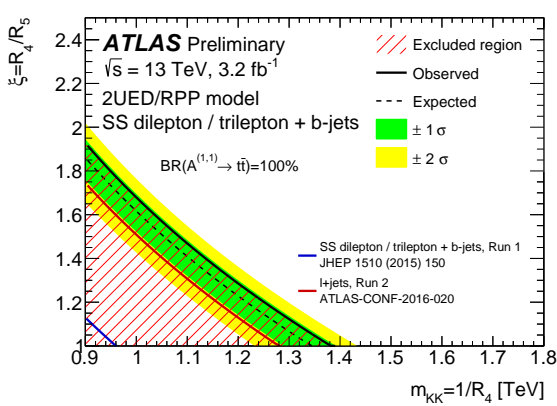

(d)

Figure 4: Combined 95\% CL limits on the considered BSM models. (a) Limit on the $T$ mass as a function of its braching ratio. (b) Limit on the $T_{5 / 3}$ mass. (c) Limit on the coupling $C_{4 t}$ versus the $\Lambda$ scale for the contact interaction model. (d) Limit on the Kaluza-Klein mass versus the ratio $R_{4} / R_{5}$ of the distance scales of the two extra dimensions.

\section{References}

[1] ATLAS Collaboration, The ATLAS Experiment at the CERN Large Hadron Collider, 2008 JINST 3 S08003.

[2] ATLAS Collaboration, Search for new physics using events with b-jets and a pair of same charge leptons in $3.2 \mathrm{fb}^{-1}$ of pp collisions at $\sqrt{\mathrm{s}}=13 \mathrm{TeV}$ with the ATLAS detector,

ATLAS-CONF-2016-032, [http://cds.cern.ch/record/2161545]

[3] J. A. Aguilar-Saavedra, R. Benbrik, S. Heinemeyer and M. Pérez-Victoria, Handbook of vectorlike quarks: Mixing and single production, Phys. Rev. D 88, 094010 (2013) [arXiv:1306.0572]

[4] M. Guchait, F. Mahmoudi and K. Sridhar, Associated production of a Kaluza-Klein excitation of a gluon with a t anti-t pair at the LHC, Phys. Lett. B 666 (2008) 347 [arXiv:0710.2234].

[5] C. Degrande, J. M. Gerard, C. Grojean, F. Maltoni and G. Servant, Non-resonant New Physics in Top Pair Production at Hadron Colliders, JHEP 1103 (2011) 125 [arXiv:1010.6304].

[6] ATLAS Collaboration, Analysis of events with b-jets and a pair of leptons of the same charge in $p p$ collisions at $\sqrt{s}=8 \mathrm{TeV}$ with the ATLAS detector, JHEP 10 (2015) 150, [arXiv:1504.04605]

[7] CMS Collaboration, Search for top quark partners with charge $5 / 3$ at $\sqrt{s}=13 \mathrm{TeV}$, CMS-PAS-B2G-15-006, [http://cds.cern.ch/record/2114805] 